\title{
Neutropénie fébrile aux urgences, stratification du risque et conditions du retour à domicile
}

\section{Febrile Neutropenia in the Emergency Department, Risk Stratification and Terms for Outpatient Management}

\author{
J. Jacquet $\cdot$ G. Catala $\cdot$ J.-P. Machiels $\cdot$ A. Penaloza
}

Reçu le 9 novembre 2018; accepté le 28 janvier 2019

(C) SFMU et Lavoisier SAS 2019

Résumé La neutropénie fébrile (NF) est une situation fréquemment rencontrée aux urgences avec un taux de mortalité non négligeable variant de 5 à $40 \%$. Cette variabilité importante met en avant l'importance de stratifier le risque afin de permettre un traitement ambulatoire per os de certains patients à faible risque. En plus du MASCC (The Multinational Association for Supportive Care in Cancer) score, d'autres outils permettent d'évaluer ce risque ou sont à l'étude dans ce but, tels que le dosage de la CRP, la procalcitonine ou encore le score CISNE. Après une prise en charge rapide aux urgences incluant l'administration sans délai d'un traitement adéquat, la poursuite de l'antibiothérapie per os à domicile est envisageable chez les patients à faible risque. La combinaison amoxicilline-acide clavulanique et ciprofloxacine est le plus souvent recommandée, mais la moxifloxacine ou la lévofloxacine en monothérapie peuvent également être utilisées pour les patients traités à domicile. Le retour à domicile permet de réduire fortement les coûts engendrés par l'hospitalisation, de diminuer le

J. Jacquet $(\bowtie)$

Université Catholique de Louvain,

1150 Bruxelles, Belgique

e-mail : jonathan.jacquet@uclouvain.be

G. Catala

Département d'oncologie et hématologie,

CHR Mons-Hainaut, groupe Jolimont, 7000 Mons, Belgique

J.-P. Machiels

Département d'oncologie, institut Roi-Albert-II,

cliniques universitaires Saint-Luc et Institute for Clinical and Experimental Research (pôle MIRO),

université catholique de Louvain, 1150 Bruxelles, Belgique

\section{A. Penaloza}

Département de médecine d'urgence,

cliniques universitaires Saint-Luc,

université Catholique de Louvain, 1150 Bruxelles, Belgique risque d'infection nosocomiale et d'améliorer la qualité de vie des patients avec NF à faible risque. Dans cette optique, plusieurs critères doivent être remplis, et une discussion avec le patient reste primordiale à la prise de décision. Parmi ceux-ci, nous retiendrons notamment un score MASCC supérieur à 21, une durée attendue de neutropénie inférieure à sept jours, l'accord du patient et de son entourage ainsi que la proximité entre le domicile et un service de soin adapté.

Mots clés Neutropénie $\cdot$ Fébrile $\cdot$ Ambulatoire $\cdot$ Risque MASCC

Abstract Febrile neutropenia is a commonly encountered complication in the emergency department (ED) with a mortality rate from 5 to $40 \%$. This significant variability highlights the importance of stratifying risk to allow oral outpatient treatment of some low-risk patients. Besides the MASCC (The Multinational Association for Supportive Care in Cancer) score, other tools allow to evaluate this risk or are under study for this purpose such as the assay of c-reactive protein (CRP), procalcitonin or the CISNE (Clinical index of stable febrile neutropenia) score. After the quick administration of an adequate treatment, the emergency physician may consider outpatient management with oral antibiotics. The combination of amoxicillin/clavulanic acid and ciprofloxacin is most often recommended but moxifloxacin or levofloxacin monotherapy may also be used for outpatient treatments. Returning home can greatly reduce the costs incurred by hospitalization, reduce the risk of nosocomial infection and improve the quality of life of patients with low-risk febrile neutropenia. To this purpose, a few criteria must be met and a talk with the patient is predominant in this decision-making process. In particular, we will consider a MASCC score $>21$, an expected duration of neutropenia $<7$ days, the agreement 
of the patient and those around him, as well as the proximity of the home to an adapted care service.

\section{Keywords Neutropenia · Febrile $\cdot$ Outpatient $\cdot$ Risk MASCC}

\section{Introduction}

La neutropénie fébrile (NF) est une complication fréquente des traitements par chimiothérapie anticancéreuse et reste, en 2018, grevée d'une mortalité importante de 5 à $18 \%$ dans nos contrées et pouvant aller jusqu'à $40 \%$ dans certaines régions [1-5].

C'est un des motifs d'admission aux urgences les plus fréquents chez les patients traités par chimiothérapie anticancéreuse.

Classiquement, le traitement consistait en une antibiothérapie intraveineuse et à une hospitalisation du patient. Le risque de NF est estimé entre 3 et $19 \%$ par cycle de chimiothérapie, représentant 60000 à 100000 hospitalisations par an aux États-Unis, dont la durée s'étend de 5 à 15 jours $[3,5,6]$. Depuis plus de 20 ans, de nombreux scores sont consacrés à évaluer le risque de complication chez les patients en NF [1,4,7-9]. Les recherches se poursuivent afin d'améliorer la pertinence de ces scores et de trouver de nouveaux facteurs pronostiques [9-15]. Ainsi, outre le diagnostic et la rapidité de prise en charge de ces patients, le rôle du médecin urgentiste implique également l'évaluation du risque de complication.

Plusieurs travaux ont montré une efficacité similaire de l'antibiothérapie orale à celle des traitements intraveineux chez les patients à faible risque de complications [16,17], ce qui a permis d'envisager, en concertation avec l'oncologue et le patient, un traitement ambulatoire [1,3,8,18-23]. Un retour à domicile, permettant une amélioration de la qualité de vie des patients ainsi qu'une réduction du risque d'infection nosocomiale et des coûts hospitaliers, est donc envisageable dans certaines conditions [18,24-26].

\section{Définition et épidémiologie}

Plusieurs définitions de la NF ont été proposées. Les plus utilisées sont celles de l'Infectious Diseases Society of America (IDSA) et de la Société européenne d'oncologie médicale (ESMO) $[1,4]$.

L'ESMO définit la NF par :

- une température orale supérieure ou égale à $38,3{ }^{\circ} \mathrm{C}$ en une prise ou supérieure à $38^{\circ} \mathrm{C}$ lors de deux prises à deux heures d'intervalle ;
- un compte absolu de neutrophile inférieur à $0,5 \times 10^{9} / 1$ ou un nadir attendu sous cette valeur [1].

L'incidence de cette complication varie énormément et dépend notamment du type de chimiothérapie et du type de cancer. En effet, beaucoup de chimiothérapies sont à faible risque d'induire une NF, c'est-à-dire moins de $10 \%$ (faible $\leq$ $10 \%$, intermédiaire $=10-20 \%$; haut $\geq 20 \%$ ). L'incidence varie également en fonction du type de cancer et peut atteindre les $80 \%$ dans certains cancers hématologiques $[5,6]$.

Malgré les progrès dans leur prise en charge, la mortalité reste élevée, variant de 5 à $40 \%$ [2-6,27]. Cette variabilité importante reflète la grande hétérogénéité de la population en termes de risque de complication. Il semble donc important de classifier ce risque et en particulier d'identifier des patients à faible risque pouvant bénéficier d'un traitement ambulatoire.

\section{Délai de prise en charge}

Afin de diminuer les risques de complications et le taux de mortalité, la prise en charge de la NF doit être rapide et l'antibiothérapie empirique administrée dans l'heure suivant l'admission $[1,19,28]$. Le risque de mortalité augmente de $18 \%$ pour chaque heure de délai avant l'administration des antibiotiques [29] et majore la durée d'hospitalisation de huit heures en moyenne [30]. La rapidité d'instauration du traitement représente un défi majeur dans nos services d'urgences surchargés. En 2010, André et al. [31] rapportaient que seulement $22 \%$ des patients (toutes catégories de risque confondues) avaient reçu leur première dose d'antibiotiques dans les 90 minutes après l'admission. Plusieurs solutions ont été proposées afin de réduire ce délai. En particulier, Keng et al. [32] en 2015 proposent une action en huit points :

- classer la NF dans l'index de classification des sévérités au même niveau qu'un AVC ou un infarctus du myocarde ;

- installer d'emblée dans des chambres les patients suspects de NF sans passer par la salle d'attente ;

- standardiser la définition de NF ;

- créer une carte d'alerte NF ;

- identifier une personne de référence dans le service pour standardiser les traitements ;

- administrer les antibiotiques avant l'obtention du compte de neutrophile ;

- disposer des antibiotiques aux urgences ;

- éduquer le personnel et les patients eux-mêmes.

Bien que certaines mesures soient discutables et limitent la validité de cette proposition, par exemple l'administration des antibiotiques avant le compte des neutrophiles, l'application de ces mesures simples a permis dans cette étude de 
réduire le délai de traitement de moitié (169 vs 81 minutes) et la durée d'hospitalisation moyenne de 2,3 jours. Cela doit inciter chaque institution, chaque service d'urgence à implémenter un protocole local de prise en charge des patients en NF.

\section{Évaluation et examens complémentaires}

La première étape est toujours la recherche de signes de gravité du sepsis tels que des signes de choc, une hypotension artérielle ou encore un qSOFA (quick Sepsis Related Organ Failure Assessment) supérieur ou égal à 2 et le traitement de ceux-ci. La prise en charge du choc n'est pas détaillée ici et ne fait pas l'objet de cet article.

Dès l'admission, une anamnèse précise et méticuleuse est conduite. Les portes d'entrée et localisations infectieuses peuvent être masquées ou absentes chez les patients en NF, en particulier chez ceux recevant des corticoïdes. Une attention marquée est portée au type de cancer, au délai depuis la dernière chimiothérapie ainsi qu'au type de chimiothérapie. La prescription prophylactique d'antibiotique, l'utilisation de facteurs de croissance hématopoïetique, les antécédents d'infection documentée, l'utilisation de corticostéroïdes, une chirurgie récente ainsi que les allergies du patient sont recherchés et mentionnés dans le dossier [1,4,28].

La recherche de foyers septiques et de métastases septiques, les facteurs de comorbidités associés (diabète, troubles neurologiques, insuffisance cardiaque, rénale ou hépatique ou encore la BPCO) sont importants à rechercher [4]. Un examen clinique rigoureux permet de prendre en compte tout changement noté par le patient, même minime, tant les signes peuvent être frustes. L'examen de la peau (y compris les points de ponction des cathéters intraveineux), de l'oropharynx, du système digestif, des poumons et du périnée doit être attentif, car souvent foyers primaires d'infection [4].

Une prise de sang complète doit être prélevée et un compte urgent des neutrophiles demandé. Deux paires d'hémocultures doivent également être obtenues avant administration de l'antibiothérapie, dont une par le cathéter veineux central si présent $[4,28]$. Les prélèvements bactériologiques multiples (frottis peau, pus, plaie, gorge...) sont réalisés en fonction des points d'appel cliniques du patient. Une analyse d'urine est souvent recommandée. En cas de diarrhées, des coprocultures sont prélevées avec notamment la recherche de Clostridium difficile. Une radiographie de thorax est réalisée au moindre doute. Les autres investigations par radiographie, échographie, scanner, IRM, ponction lombaire, lavage bronchoalvéolaire sont laissées à l'appréciation du clinicien. Le tableau 1 présente les différents examens paracliniques envisageables.

\section{Évaluation du risque}

Jusqu'au début des années 2000, la majorité des NF étaient traitées par antibiothérapie intraveineuse avec nécessité d'une hospitalisation du patient [19]. La durée d'hospitalisation moyenne était de 5 à $15 \mathrm{j}$ et représentait un coût important (7 825 à $20117 €$ par hospitalisation). La volonté de

\begin{tabular}{|c|c|c|c|}
\hline Laboratoire & Prélèvements microbiologiques & Radiologie & $\begin{array}{l}\text { Autres (en fonction } \\
\text { de la clinique) }\end{array}$ \\
\hline $\begin{array}{l}\text { Formule sanguine complète } \\
\text { avec compte des leucocytes } \\
\text { et plaquettes }\end{array}$ & $\begin{array}{l}2 \text { paires d'hémoculture : } 1 \text { en } \\
\text { périphérie et } 1 \text { sur chaque lumière } \\
\text { d'un cathéter à demeure ou } 2 \\
\text { périphériques si pas de cathéter }\end{array}$ & $\begin{array}{l}\text { Radiographie de thorax si } \\
\text { symptôme ou signe } \\
\text { respiratoire }\end{array}$ & Lavage bronchoalvéolaire \\
\hline Urée et créatinine & Urine si nécessaire & $\begin{array}{l}\text { Échographie ou scanner } \\
\text { en fonction de la clinique }\end{array}$ & $\begin{array}{l}\text { Gazométrie artérielle si } \\
\text { nécessaire }\end{array}$ \\
\hline $\begin{array}{l}\text { Électrolytes (sodium, potassium, } \\
\text { chlore, bicarbonate...) }\end{array}$ & Expectorations si nécessaire & & Échographie cardiaque \\
\hline $\begin{array}{l}\text { Fonction hépatique } \\
\text { (transaminases, bilirubine...) }\end{array}$ & Prélèvement de gorge si nécessaire & & \\
\hline Lactate & Prélèvements de plaie si nécessaire & & \\
\hline Hémostase & $\begin{array}{l}\text { Coprocultures si nécessaire } \\
\text { et recherche de Clostridium difficile }\end{array}$ & & \\
\hline $\begin{array}{l}\text { Marqueurs inflammatoires } \\
\text { et infectieux (CRP, fibrinogène) }\end{array}$ & & & \\
\hline
\end{tabular}


réduire le temps de séjour hospitalier pour ces patients, afin d'améliorer leur qualité de vie, de réduire le risque d'infection nosocomiale ainsi que les coûts engendrés, explique la littérature abondante à la recherche de moyens d'identification de patients à faible risque pouvant bénéficier d'un traitement ambulatoire [1,4,6,8,19,33].

L'importance de l'évaluation du risque est soulignée dans toutes les recommandations internationales. De très nombreuses études se sont intéressées à la catégorisation du risque de complication et de mortalité des patients en NF.

Une des premières règles proposées fut celle de Talcott classant en quatre catégories de risque les patients en NF, rapidement remplacée par le score MASCC (The Multinational Association for Supportive Care in Cancer), plus performant (Tableau 2) [7]. De nombreuses études ont évalué et validé le score MASCC, montrant des valeurs prédictives positives supérieures à $83 \%$ et une sensibilité entre 59 et $95 \%$ contre seulement $30 \%$ de sensibilité pour le modèle de Talcott [7]. Dans la plupart des recommandations internationales, le score MASCC $(>21)$ est mentionné pour identifier les patients à faible risque $[1,4,8,19,28,33]$ avec une probabilité de complication sévère de l'ordre de $6 \%$ et de mortalité inférieure à $1 \%$ [1].

Une récente revue de la littérature valide la place du score MASCC pour les cancers solides et hématologiques mais pointe également certaines faiblesses du modèle [7]. Premièrement, le modèle n'inclut pas les principales comorbidités telles que le diabète, le statut mental altéré, l'insuffisance cardiaque... Les auteurs estiment que ces comorbidités peuvent être reprises dans l'item « Burden of Illness » [7]. Ce

Tableau 2 Score MASCC : the Multinational Association for Supportive Care in Cancer (adapté de Badertscher et al. [28])

\begin{tabular}{|c|c|}
\hline Caractéristiques & Points \\
\hline $\begin{array}{l}\text { Sévérité du tableau clinique initial : symptômes } \\
\text { absents ou légers }\end{array}$ & 5 \\
\hline $\begin{array}{l}\text { Sévérité du tableau clinique initial : symptômes } \\
\text { modérés }\end{array}$ & 3 \\
\hline Absence d'hypotension & 5 \\
\hline $\begin{array}{l}\text { Absence de bronchopneumopathie chronique } \\
\text { obstructive (BPCO) }\end{array}$ & 4 \\
\hline $\begin{array}{l}\text { Tumeur solide ou hémopathie maligne } \\
\text { en l'absence d'infection fongique préalable }\end{array}$ & 4 \\
\hline Absence de déshydratation & 3 \\
\hline $\begin{array}{l}\text { Patient ambulatoire au moment de l'apparition } \\
\text { de la fièvre }\end{array}$ & 3 \\
\hline$\hat{\text { Âge }}<60$ ans & 2 \\
\hline
\end{tabular}

point est en effet une des difficultés pour établir ce score, car il n'existe pas de définition claire de cet item. Celui-ci pourrait être traduit en français par la lourdeur, le poids de la maladie. Deuxièmement, le modèle ne prend pas en compte la durée attendue de la neutropénie, or si cette durée attendue dépasse les sept jours, la plupart des auteurs et les recommandations de l'IDSA classent le patient à haut risque d'emblée [4]. Ces raisons expliquent les nombreuses tentatives d'amélioration du score MASCC et de validation d'un nouveau score plus performant.

En 2014, dans une étude rétrospective, Elhan [15] tente d'identifier des facteurs indépendants de mauvais pronostic. Parmi ceux prédictifs de mortalité, on note des plaquettes inférieures à $50000 / \mathrm{mm}^{3}$ (également retrouvé dans d'autres études $[34,35])$, la présence d'un infiltrat à la radiographie de thorax, une hypoprotéinémie inférieure à $6 \mathrm{~g} / \mathrm{dl}$, une fréquence respiratoire supérieure à $24 /$ minute et un score MASCC inférieur à 21 [15]. Yapici et al. [36], en 2016, identifient eux aussi des critères délétères indépendants : un score MASCC inférieur à 21 ainsi qu'une neutropénie prolongée.

En 2017, Wang et al. [37] évaluent la performance pronostique de l'association de la perception de santé du patient par le FACT-N questionnaire (Functional Assessment of Cancer Therapy-Neutropenia) au score MASCC pour identifier les patients à faible risque de complication. Les auteurs dérivaient un score en pondérant le MASCC et le résultat de l'échelle "malaise » du FACT-N. Ils concluent que les patients ayant une meilleure perception de santé selon l'échelle «malaise » associée à un score MASCC supérieur à 21 étaient moins à risque de complication [37]. Malgré les limitations de l'étude (faible nombre de patients, cancer solide...) et la difficulté de généralisation du score de perception du patient (celle-ci varie en fonction du lieu [Chine], de la culture et de l'environnement...), cette étude est une des premières à intégrer la perception du patient de sa propre situation clinique dans le processus de catégorisation du risque.

En 2011, Carmona-Bayonas et al. [38], dans une population de patients adultes hémodynamiquement stables dans les trois heures suivant l'admission, avec tumeur solide, chimiothérapie de faible ou moyenne intensité et traités en ambulatoire, cherchent à identifier des facteurs de mauvais pronostic. Les critères d'exclusion comprenaient l'absence de dysfonction d'organe (rénal, cardiaque et respiratoire) et de décompensation d'une insuffisance chronique d'organe, l'absence d'infection sévère documentée et l'absence de complication sévère autre justifiant une hospitalisation. Les auteurs proposent ainsi un nouveau score, le score CISNE (Clinical Index of Stable Febrile Neutropenia) (Tableau 3). Ce score, validé dans une étude prospective multicentrique, propose une classification en trois classes : classe 1 ( 0 point), classe 2 ( 1 à 2 points) et classe 3 ( $>2$ points). Les taux de 
Tableau 3 Score CISNE : index clinique de neutropénie fébrile stable (adapté de Carmona-Bayonas et al. [39])

\begin{tabular}{|ll|}
\hline Caractéristiques & Points \\
\hline Score ECOG PS $\geq 2$ & 2 \\
Hyperglycémie & 2 \\
BPCO & 1 \\
Maladie cardiovasculaire chronique & 1 \\
Mucite NCI $\geq 2$ & 1 \\
Monocytes $<200 / \mu \mathrm{l}$ & 1 \\
\hline
\end{tabular}

complications et de mortalité sont respectivement de 1,1 et $0 \%$ pour la classe 1 ; de 6,2 et $0 \%$ pour la classe 2 et de 36 et $3,1 \%$ pour la classe 3 [39]. On note que la mortalité reste très faible dans l'étude de validation du score même parmi les patients de la classe 3 , supposée la plus à risque. L'intérêt majeur de ce score est de proposer une catégorisation plus fine des patients à faible risque. Parmi les patients à faible risque (selon MASCC), hémodynamiquement stables et sans comorbidité majeure, le score CISNE pourrait identifier un sous-groupe à risque très faible pouvant être aisément traité en ambulatoire et un sous-groupe à risque plus élevé qui pourrait bénéficier d'une hospitalisation [39]. En 2017, une étude rétrospective comparant les scores MASCC et CISNE montre une spécificité nettement supérieure du CISNE pour identifier les patients à faible risque $(98,3$ vs $54,2 \%$ [ [9]. Cependant, cette étude comporte des limitations :

- les patients avec tumeurs solides et hématologiques ainsi que des patients non stables à l'admission ou avec des critères d'exclusion pour le score CISNE ont tout de même été enrôlés dans l'étude ;

- seulement quatre patients ont été traités ambulatoirement et, parmi les autres, une durée d'hospitalisation de trois jours pour les faibles risques selon CISNE comparée à six jours pour les hauts risques a été retrouvée. Des études supplémentaires sont à ce stade nécessaires pour valider ce score.

Un autre facteur de risque étudié est la présence d'une bactériémie lors de l'admission, son incidence étant élevée variant de 22 à $32 \%$ [2]. De nombreuses études ont montré une mortalité majorée chez les patients avec bactériémie (10 vs $3 \%$ ) ainsi qu'un taux de complication plus important (31 vs $14 \%$ ). Notons qu'aucune différence statistiquement significative n'était retrouvée entre les patients avec tumeur solide versus hématologique [2]. Une bactériémie à Gram négatif montre également des taux de mortalité plus élevée comparée à une bactériémie à Gram positif ( 18 à $47 \%$ versus 5 à $16 \%)[2,27]$.

L'importance de la détection précoce de bactériémie a suscité l'intérêt pour plusieurs biomarqueurs comme la pro- calcitonine (PCT), les interleukines (IL) 6 et 8 et la protéine $\mathrm{C}$ réactive (CRP).

Les IL 6 et 8 semblent présenter un intérêt dans la classification du risque de développer une infection sévère et leur détection et pourraient permettre de distinguer un groupe de patients avec NF de faible durée et à faible risque d'infection sévère, d'un groupe de patients avec infection sévère ou sepsis à hémocultures positives $[8,40]$. Un dosage régulier tous les trois à quatre jours de l'IL 6 chez les patients traités pour un cancer du sein, afin de détecter une élévation du marqueur, pourrait être un prédicteur de l'apparition d'une NF [10]. Dans la même étude, le dosage de CRP atteint un pic au moment de l'apparition de la NF et est donc de peu d'intérêts en termes de prédiction de la survenue d'une NF, mais pourrait être utile dans l'évaluation du risque d'infection sévère [10].

La PCT et la CRP semblent prometteuses. La PCT a montré un intérêt dans la détection des infections sévères. Ainsi, un dosage de PCT supérieur à $2 \mathrm{ng} / \mathrm{ml}$ suggèrerait une infection sévère ou un choc septique, alors qu'un dosage inférieur à $0,5 \mathrm{ng} / \mathrm{ml}$ identifierait des patients à faible risque d'infection sévère [11]. Cependant, ce faible taux de PCT n'exclut pas les phases précoces d'infection fungique, virale et est retrouvé dans les fièvres d'origine indéterminée (FUO) [11]. Une des difficultés dans cette revue de littérature est la grande variabilité des tests pour le dosage de la PCT qui ont évolué depuis (Tableau 4).

En 2013, Ahn et al. [13] montrent qu'un score MASCC inférieur à 21, un compte de plaquettes inférieur à 100 000/ $\mathrm{mm}^{3}$ et un dosage de PCT supérieur ou égal à $0,5 \mathrm{ng} / \mathrm{ml}$ sont des facteurs indépendants de bactériémie. Le score MASCC a été construit pour identifier des patients à faible risque de complication et non pour détecter la présence de bactériémie [13]. La combinaison PCT supérieure ou égale à $5 \mathrm{ng} / \mathrm{ml} \mathrm{au}$ score MASCC inférieur à 21 augmenterait la spécificité de détection d'une bactériémie (90 à $94 \%$ ) ou d'un choc septique (90 à $98 \%$ ), au prix d'une diminution importante de la sensibilité du test (46 à $37 \%$ ). Parmi les 306 patients à faible risque (MASCC > 21), l'association d'un taux de PCT inférieur à $0,5 \mathrm{ng} / \mathrm{ml}$ résulte en un taux de faux-négatifs de $2,8 \%$ pour la bactériémie [13].

L'intérêt des biomarqueurs dans l'évaluation du risque semble donc réel, mais aucun d'entre eux n'a été validé prospectivement et internationalement actuellement.

Enfin, la plupart des recommandations proposent de traiter comme des hauts risques, les patients présentant des comorbidités importantes, une dysfonction d'organes ou, en cas d'infection sévère, telle qu'une pneumonie, une cellulite, un autre site bien défini d'infection, une infection d'un cathéter intraveineux, les patients en insuffisance rénale ou hépatique, ceux qui présentent une douleur abdominale nouvelle ou encore un statut neurologique modifié, une intolérance orale et ceux présentant des vomissements incoercibles $[1,4,19]$. 


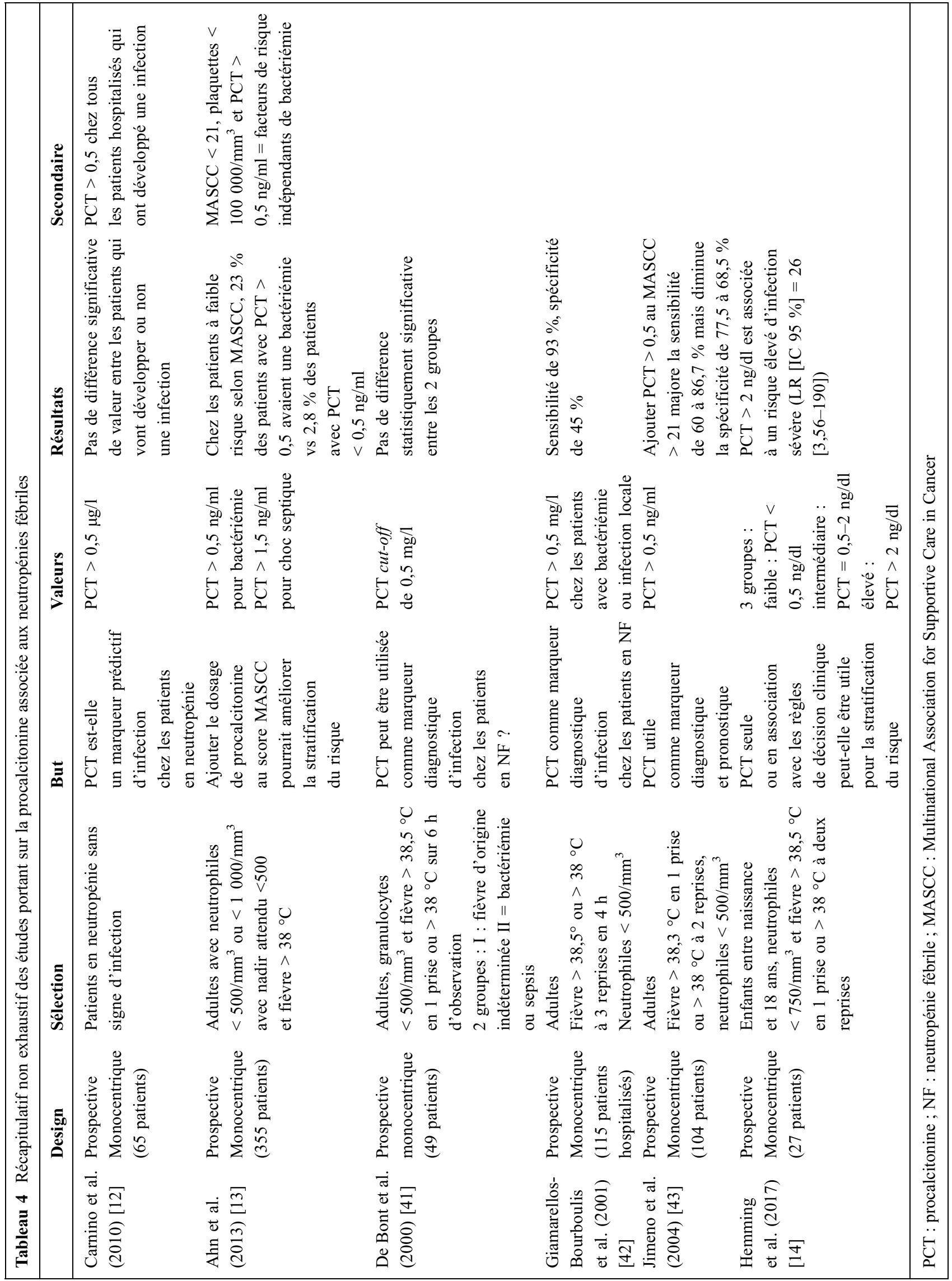




\section{Bactériémie, changement des micro- organismes responsables et résistances}

Historiquement, les premières bactéries isolées lors des bactériémies chez les patients en NF étaient des Gram négatif [44]. Depuis une vingtaine d'années, on note une majoration des bactéries à Gram positif. Cela s'explique notamment par l'utilisation plus importante des cathéters à demeure centraux et périphériques qui sont le siège de colonisation par ces bactéries [44]. Récemment, une tendance à la résurgence des bactériémies à Gram négatif a été notée $[44,45]$. Plusieurs explications sont avancées à ce nouveau changement, parmi lesquelles on retrouve la diminution de l'usage d'antibioprophylaxie, notamment par fluoroquinolone $[44,46]$. Le type de chimiothérapie peut également être incriminé, l'arrivée d'agents moins cytotoxiques a diminué l'incidence de mucites sévères et entraîne des neutropénies moins profondes, résultant en une diminution des streptocoques viridans et entérocoques [44]. La diminution de l'utilisation des cathéters à demeure a également réduit les colonisations et donc les bactériémies à Gram positif.

Les bactéries à Gram négatif le plus souvent rencontrées comprennent Escherichia coli, Pseudomonas aeruginosa et Klebsiella pneumoniae $[44,45]$. Les bactéries à Gram positif les plus fréquentes sont quant à elles les streptocoques, staphylocoques et entérocoques [44].

On observe également depuis quelques années l'apparition de bactéries résistantes, telles que les bactéries produisant des bêtalactamases à spectre élargi et semblant être responsables d'une élévation des taux de mortalité, d'admission aux soins intensifs et d'aggravation de l'évolution du patient $[44,45]$. D'autres résistances sont à noter comme celle d'entérocoques résistant à la vancomycine liés à une majoration importante de la mortalité.

Il n'existe pas, à l'heure actuelle, d'évidence causale entre l'utilisation de fluoroquinolone prophylactique et la survenue d'infection ou de colonisation par des germes résistants aux quinolones [44-46]. La majoration de résistance dans la population souffrant de cancer est également à mettre en lien avec la majoration des résistances dans la population générale [44].

Le résultat des prélèvements est souvent retardé par rapport à l'admission du patient, mais si une bactériémie est détectée et le patient retourné au domicile, celui-ci doit être rappelé pour éventuellement être hospitalisé selon la bactériémie retrouvée.

\section{Antibiothérapie}

\section{Haut risque}

L'antibiothérapie empirique est instaurée le plus rapidement possible après l'admission du patient. Les recommandations proposent d'instaurer une antibiothérapie empirique à large spectre chez les patients à haut risque et insistent sur la connaissance de l'épidémiologie locale et la distribution des résistances médicamenteuses pour choisir au mieux le traitement $[1,4,19,33]$.

Il est recommandé de commencer un traitement empirique en monothérapie par une bêtalactamine à spectre élargi active contre le pseudomonas comme le céfépime, par une carbapénème (imipénem-cilastatine, méropénèm) ou par pipéracilline-tazobactam $[1,4,33]$. Une récente métaanalyse compare ces traitements empiriques par bêtalactamines actives contre le pseudomonas regroupant 50 études et 10872 patients. Celle-ci montre une légère supériorité de l'imipénem-cilastatine en termes de mortalité toute cause confondue et montre une infériorité de la ceftazidime en termes de succès du traitement sans nécessité de changement au cours de l'hospitalisation [47]. Les auteurs concluent que l'imipénem-cilastatine, le pipéracilline-tazobactam et le méropénèm sont des premiers choix raisonnables pour le traitement empirique de la NF à haut risque. En cas de réaction d'hypersensibilité immédiate à la pénicilline, il est recommandé d'éviter les bêtalactamines et les carbapénèmes et d'utiliser une association de type ciprofloxacine-clindamycine ou aztréonam-vancomycine [4].

L'association de la vancomycine dans la prise en charge initiale n'est pas recommandée. Elle est proposée en seconde intention, si nécessaire, dans des cas particuliers tels que les infections de cathéter, les pneumonies, les infections des tissus mous et de la peau et chez les patients instables hémodynamiquement [4]. Si le patient est à risque de contracter une infection à germe résistant de type staphylocoque doré résistant à la méticilline, entérocoque résistant à la vancomycine ou bactérie productive de betalactamases à spectre élargi, il est recommandé d'ajouter un second agent antiinfectieux en combinaison.

\section{Risques faibles}

Chez les patients à faible risque de complication et de mortalité, un traitement ambulatoire peut être envisagé. L'équivalence d'un traitement oral versus intraveineux de l'antibiothérapie dans la NF à faible risque a été largement démontrée $[1,4,8,16]$.

Une combinaison d'amoxicilline/acide clavulanique avec de la ciprofloxacine est le plus souvent recommandée $[1,4,8,19,28,33]$. L'association clindamycine-ciprofloxacine peut être envisagée comme alternative chez les patients présentant une réaction d'hypersensibilité à l'amoxicillineacide clavulanique [4,19]. L'utilisation d'une fluoroquinolone à large spectre peut également être une alternative. La moxifloxacine et la lévofloxacine en monothérapie peuvent être utilisées même si des études à plus large échelle sont nécessaires $[4,8,17,48,49]$. L'utilisation de ciprofloxacine 
seule n'est par contre pas recommandée [4]. Les patients ayant reçu une prophylaxie antibiotique par fluoroquinolones ne peuvent pas être traités avec une quinolone et sont en général classés d'emblée à haut risque $[1,4,8]$.

\section{Retour à domicile}

Les recommandations récentes proposent un traitement ambulatoire des patients ayant une tumeur solide, à faible risque, dans des circonstances bien précises. Une revue de littérature de 2014 suggère la même attitude pour les patients avec un cancer hématologique [33]. Dans cette revue, Villafuerte-Gutierrez et al. [33] revisitent les guidelines existant pour évaluer le risque. Ils proposent donc d'utiliser le score MASCC supérieur à 21 pour classer en faible risque, mais également la durée attendue de neutropénie inférieure à sept jours, l'absence de comorbidité importante et l'absence d'insuffisances rénale et hépatique. D'autres critères peuvent aussi être ajoutés (Tableau 5) [33].

Pour les recommandations ESMO, un traitement ambulatoire peut être proposé chez les patients évalués à faible risque après une période d'observation de 24 heures [1]. L'ASCO et l'IDSA ne précisent pas de durée d'observation, et il est à noter que peu d'études ont proposé un retour à domicile sans période d'observation, celle-ci variant de 2 à 72 heures $[4,19]$.

L'ASCO recommande, sur base de l'opinion d'experts, une période d'observation d'au moins quatre heures. Dans tous les cas, la fièvre doit être documentée, les prélèvements bactériologiques réalisés, la première dose d'antibiotique donnée le plus rapidement, la stabilité clinique du patient observée et la tolérance à la prise orale de l'antibiotique vérifiée avant la sortie du patient [19]. Le succès du traitement empirique est élevé (de 80 à plus de $90 \%$ ), sans différence significative entre les traitements ambulatoire et hospitalier $[4,19]$.

En 2011, une méta-analyse montre une efficacité comparable en termes de mortalité (risque relatif [RR] : 1,11 ; intervalle de confiance à $95 \%$ [IC $95 \%]:[0,41-3,05]$; $p=0,83$ ) et d'échec de traitement (RR : 0,81 ; IC $95 \%$ : $[0,55-1,19] ; p=0,28)$, du traitement ambulatoire par rap- port au traitement intrahospitalier de patients en NF à faible risque [22]. On note plusieurs limitations à cette métaanalyse : les critères d'exclusion n'étaient pas identiques dans les deux groupes (tumeur hématologique, transplantation de cellule souche, site d'infection déterminé...), le choix de l'antibiothérapie initiale était très hétérogène, de même pour le délai d'administration de la première dose d'antibiotique et le délai d'observation avant sortie [22]. En 2011, une étude randomisée multicentrique prospective confirme l'efficacité similaire entre un traitement intrahospitalier vs extrahospitalier chez les patients à faible risque, avec des taux de complications identiques dans les deux groupes (9 vs $8 \% ; p=0,56)$ [50]. En 2014, une revue de littérature a évalué la sécurité d'un retour précoce à domicile directement depuis le service des urgences. Aucune différence n'est observée entre les deux groupes : mortalité de 0 à $4 \%$ en ambulatoire contre 0 à $2 \%$ en intrahospitalier, taux de succès de l'antibiothérapie initiale de 77 à $91 \%$ en hospitalier contre 77 à 89,5\% en ambulatoire [21]. Cependant, 17 à $21 \%$ des patients traités en ambulatoire nécessitent une hospitalisation secondaire soit pour échec de la prise en charge ambulatoire (persistance de fièvre, intolérance à la prise orale...), soit pour hémocultures positives [21]. Bien qu'il existe de nombreuses limitations à cette revue, notamment le faible nombre d'études et de patients inclus et l'hétérogénéité des prises en charge, elle montre qu'un retour à domicile depuis le service des urgences est possible. Bitar [51] étudie, en 2015, la possibilité d'utiliser le score MASCC comme seul outil pour décider d'un traitement ambulatoire. Au seuil de 21, la sensibilité du MASCC de $94 \%$ comme critère pour ne pas hospitaliser est insuffisante pour les auteurs. Ils proposent d'y ajouter un critère supplémentaire non repris initialement dans le MASCC : l'impossibilité de manger ou d'avaler [51]. Dans cette étude, $84 \%$ des patients avec incapacité de manger/d'avaler et un score MASCC supérieur ou égal à 21 présentent une autre complication médicale et restent hospitalisés plus de neuf contre sept jours pour ceux capables de manger. Cette proposition pleine de bon sens a également été faite par d'autres auteurs [22] et se retrouve aussi dans les recommandations de l'ASCO. Certains auteurs suggèrent la possibilité de

Tableau 5 Évaluation initiale du risque pour les patients en neutropénie fébrile (adapté de Kuderer et al. [3])

\begin{tabular}{ll} 
Faible risque (score MASCC $>\mathbf{2 1})$ ou : & Haut risque (score MASCC $<\mathbf{2 1 )}$ ou : \\
\hline Patient externe à l'apparition de la fièvre & Patient hospitalisé à l'apparition de la fièvre \\
Absence de comorbidité & Comorbidité importante ou instabilité clinique \\
Durée de neutropénie attendue $<7 \mathrm{j}$ & Durée de neutropénie attendue $>7 \mathrm{j}$ \\
Statu ECOG $0 / 1$ & Insuffisance rénale ou hépatique \\
Absence d'insuffisance rénale et hépatique & Mucites de grades $3-4$
\end{tabular}

MASCC : Multinational Association of Supportive Care in Cancer ; ECOG : Eastern Cooperative Oncolgy Group Performance Status 
Tableau 6 Critères requis pour envisager un traitement ambulatoire (adapté de Kridel et al. [49])

\section{Critères pour envisager un traitement ambulatoire des patients en neutropénie fébrile à faible risque de complications}

Score MASCC $\geq 21$, neutropénie attendue d'une durée de moins de $7 \mathrm{j}$ et absence de neutropénie extrême $\left(<100\right.$ neutrophiles $\left./ \mathrm{mm}^{3}\right)$ Paramètres cliniques stables aux urgences pendant une observation de 4 à $6 \mathrm{~h}$ minimum

Accord du patient et de son entourage

Structure de soins hospitalière adéquate à moins de $1 \mathrm{~h}$ du lieu de résidence

Absence de prophylaxie par fluoroquinolone

Disponibilité du médecin traitant et d'un service infirmier à domicile pour des soins quotidiens

Compréhension des complications possibles et établissement de critères d'alerte pour éventuelle réadmission

MASCC : Multinational Association for Supportive Care in Cancer

traiter des patients ayant des difficultés de déglutition en ambulatoire par antibiothérapie intraveineuse [7], on peut cependant craindre une perte du bénéfice du traitement extrahospitalier, une majoration du coût et un risque majoré de réadmission chez ces patients. Une prise en charge ambulatoire ne peut être envisagée que si un suivi adéquat et rapproché est organisé. Cela peut constituer un facteur limitant, notamment dans les milieux ruraux où les délais d'accès à un centre de référence peuvent être longs. Un suivi quotidien doit être proposé à ces patients, ainsi que des instructions claires fournies en cas de fièvre persistante, d'aggravation des symptômes ou de toute autre complication [7]. Des équipes mobiles se rendant au domicile existent à cet effet et peuvent même réaliser un suivi des PICC Line (cathéter central à insertion périphérique). Un retour précoce à domicile avec un traitement ambulatoire permettrait d'améliorer la qualité de vie des patients et de diminuer le risque d'infection nosocomiale, mais aussi de diminuer les coûts de la NF. Plusieurs études ont montré une réduction importante du coût du traitement sans diminution de l'efficacité ni de la sécurité pour les patients traités en ambulatoire. Une étude incluant 712 patients ayant une tumeur solide et un faible risque de complication a montré une réduction du coût d'environ $50 \%$ (6 786 versus $13298 €$ ) [18]. Hendricks et al. [25] montrent également une diminution de $49 \%$ des coûts (14 265 versus $9585 €$ ), sans majoration des coûts indirects pour le patient et ses proches, mais surtout sans majoration de la charge mentale, physique et économique des soignants informels. Le rapport coût/efficacité de quatre types de prise en charge différents pour les faibles risques a été évalué par Teuffel et al. [26] : traitement intrahospitalier avec antibiothérapie intraveineuse, libération rapide après 48 heures d'observation suivi par un traitement ambulatoire per os, traitement intraveineux ambulatoire et traitement per os ambulatoire. On retrouve une supériorité de coût/efficacité dans les prises en charge ambulatoires, sans différence entre les deux propositions ambulatoires [26]. Ainsi, un traitement ambulatoire intraveineux ou per os peut être envisagé de façon identique sur le plan financier et sans diminuer l'efficacité à condition que l'infrastructure adéquate existe.
L'amélioration de la qualité de vie du patient a aussi été documentée. La qualité de vie attendue liée à la santé a été évaluée entre ces quatre mêmes stratégies de prise en charge : intrahospitalier, extrahospitalier après un temps d'observation, ambulatoire avec antibiothérapie intraveineuse et ambulatoire avec antibiothérapie per os. Le traitement ambulatoire était préféré par $75 \%$ des patients (36\% oral, $21 \%$ intraveineux et $18 \%$ décharge rapide). L'étude va plus loin en concluant que les patients seraient prêts à sacrifier neuf à dix semaines de survie $(<1 \%$ de leur espérance de vie attendue) et payer un supplément (de l'ordre de $250 €$ ) pour éviter une hospitalisation [24]. Ces chiffres renforcent la nette préférence de la majorité des patients à être traités si possible en ambulatoire. Il faut néanmoins tenir compte de la préférence de $25 \%$ d'entre eux à rester en milieu hospitalier, probablement plus rassurant. Il est donc primordial de dialoguer avec le patient et de tenir compte de sa perception. Le tableau 6 résume les critères nécessaires pour envisager un retour à domicile dans de bonnes conditions.

\section{Conclusion}

La prise en charge d'un patient en NF doit être rapide et efficace. Après le diagnostic, le clinicien doit évaluer le risque de complications en s'aidant d'outils tels que le score MASCC, le score CISNE et d'autres facteurs indépendants de mauvais pronostic et instaurer un traitement antibiotique adéquat. Un retour à domicile pour les patients à faible risque peut être discuté après une période d'observation de stabilité clinique de minimum quatre heures, étant donné l'efficacité comparable des traitements ambulatoires et hospitaliers chez ces patients, et ne peut être proposé que si une infrastructure adéquate existe, en tenant compte des préférences du patient.

Liens d'intérêts : les auteurs déclarent ne pas avoir de liens d'intérêts. 


\section{Références}

1. Klastersky J, de Naurois J, Rolston K, et al (2016) Management of febrile neutropaenia: ESMO Clinical Practice Guidelines. Ann Oncol 27:v111-v8

2. Klastersky J, Ameye L, Maertens J, et al (2007) Bacteraemia in febrile neutropenic cancer patients. Int $\mathrm{J}$ Antimicrob Agents 30:51-9

3. Kuderer NM, Dale DC, Crawford J, et al (2006) Mortality, morbidity, and cost associated with febrile neutropenia in adult cancer patients. Cancer 106:2258-66

4. Freifeld AG, Bow EJ, Sepkowitz KA, et al (2011) Clinical practice guideline for the use of antimicrobial agents in neutropenic patients with cancer: 2010 update by the Infectious Diseases Society of America. Clin Infect Dis 52:e56-e93

5. De Castro CarpeñO J, GascóN-Vilaplana P, Tejerina AMCFD, et al (2015) Epidemiology and characteristics of febrile neutropenia in oncology patients from Spanish tertiary care hospitals: PINNACLE study. Mol Clin Oncol 3:725-9

6. Weycker D, Barron R, Kartashov A, et al (2014) Incidence, treatment, and consequences of chemotherapy-induced febrile neutropenia in the inpatient and outpatient settings. J Oncol Pharm Pract 20:190-8

7. Klastersky J, Paesmans M (2013) The Multinational Association for Supportive Care in Cancer (MASCC) risk index score: 10 years of use for identifying low-risk febrile neutropenic cancer patients. Support Care Cancer 21:1487-95

8. Klastersky J, Awada A, Paesmans M, Aoun M (2011) Febrile neutropenia: a critical review of the initial management. Crit Rev Oncol Hematol 78:185-94

9. Coyne CJ, Le V, Brennan JJ, et al (2017) Application of the MASCC and CISNE risk-stratification scores to identify lowrisk febrile neutropenic patients in the emergency department. Ann Emerg Med 69:755-64

10. Netterberg I, Karlsson MO, Nielsen EI, et al (2018) The risk of febrile neutropenia in breast cancer patients following adjuvant chemotherapy is predicted by the time course of interleukin- 6 and C-reactive protein by modelling: IL- 6 and CRP as predictors of febrile neutropenia. Br J Clin Pharmacol 84:490-500

11. Sakr Y, Sponholz C, Tuche F, et al (2008) The role of procalcitonin in febrile neutropenic patients: review of the literature. Infection 36:396-407

12. Carnino L, Betteto S, Loiacono M, et al (2010) Procalcitonin as a predictive marker of infections in chemoinduced neutropenia. J Cancer Res Clin Oncol 136:611-5

13. Ahn S, Lee YS, Lim KS, Lee JL (2013) Adding procalcitonin to the MASCC risk-index score could improve risk stratification of patients with febrile neutropenia. Support Care Cancer 21:2303-8

14. Hemming V, Jakes AD, Shenton G, Phillips B (2017) Prospective cohort study of procalcitonin levels in children with cancer presenting with febrile neutropenia. BMC Pediatr 17:2

15. Elhan AH (2014) Independent factors for prediction of poor outcomes in patients with febrile neutropenia. Med Sci Monit 20:1826-32

16. Vidal L, Ben Dor I, Paul M, et al (2013) Oral versus intravenous antibiotic treatment for febrile neutropenia in cancer patients. Cochrane Database Syst Rev 10:CD003992

17. He L, Zhou C, Zhao S, et al (2015) Once-daily, oral levofloxacin monotherapy for low-risk neutropenic fever in cancer patients: a pilot study in China. Anticancer Drugs 26:359-62

18. Elting LS, Lu C, Escalante CP, et al (2008) Outcomes and cost of outpatient or inpatient management of 712 patients with febrile neutropenia. J Clin Oncol 26:606-11

19. Flowers CR, Seidenfeld J, Bow EJ, et al (2013) Antimicrobial prophylaxis and outpatient management of fever and neutropenia in adults treated for malignancy: American Society of Clinical Oncology clinical practice guideline. J Clin Oncol 31:794-810

20. Delebarre M, Tiphaine A, Martinot A, Dubos F (2016) Riskstratification management of febrile neutropenia in pediatric hematology-oncology patients: results of a French nationwide survey. Pediatr Blood Cancer 63:2167-72

21. Mamtani M, Conlon LW (2014) Can we safely discharge lowrisk patients with febrile neutropenia from the emergency department? Ann Emerg Med 63:48-51

22. Teuffel O, Ethier MC, Alibhai SMH, et al (2011) Outpatient management of cancer patients with febrile neutropenia: a systematic review and meta-analysis. Ann Oncol 22:2358-65

23. Orme LM, Babl FE, Barnes C, et al (2014) Outpatient versus inpatient IV antibiotic management for pediatric oncology patients with low risk febrile neutropenia: a randomised trial: outpatient management of febrile neutropenia. Pediatr Blood Cancer 61:1427-33

24. Teuffel O, Cheng S, Ethier MC, et al (2012) Health-related quality of life anticipated with different management strategies for febrile neutropenia in adult cancer patients. Support Care Cancer 20:2755-64

25. Hendricks AM, Loggers ET, Talcott JA (2011) Costs of home versus inpatient treatment for fever and neutropenia: analysis of a multicenter randomized trial. J Clin Oncol 29:3984-9

26. Teuffel O, Amir E, Alibhai S, et al (2011) Cost effectiveness of outpatient treatment for febrile neutropaenia in adult cancer patients. Br J Cancer 104:1377-83

27. Horasan ES, Ersoz G, Tombak A, et al (2011) Bloodstream infections and mortality-related factors in febrile neutropenic cancer patients. Med Sci Monit 17(5):CR304-9

28. Badertscher DL, Damak H, Mercier L, Décoster D (2016) Prise en charge de la neutropénie fébrile. Rev Med Suisse 12:1321-5

29. Rosa RG, Goldani LZ (2014) Cohort study of the impact of time to antibiotic administration on mortality in patients with febrile neutropenia. Antimicrob Agents Chemother 58:3799-803

30. Perron T, Emara M, Ahmed S (2014) Time to antibiotics and outcomes in cancer patients with febrile neutropenia. BMC Health Serv Res 14:162

31. André S, Taboulet P, Elie C, et al (2010) Febrile neutropenia in French emergency departments: results of a prospective multicentre survey. Crit Care 14:R68

32. Keng MK, Thallner EA, Elson P, et al (2015) Reducing time to antibiotic administration for febrile neutropenia in the emergency department. J Oncol Pract 11:450-5

33. Villafuerte-Gutierrez P, Villalon L, Losa JE, Henriquez-Camacho C (2014) Treatment of febrile neutropenia and prophylaxis in hematologic malignancies: a critical review and update. Adv Hematol 2014:1-9

34. Moon JM, Chun BJ (2009) Predicting the complicated neutropenic fever in the emergency department. Emerg Med J 26:802-6

35. Lynn JJ, Chen KF, Weng YM, Chiu TF (2013) Risk factors associated with complications in patients with chemotherapy-induced febrile neutropenia in emergency department: Antibiotics for emergent febrile neutropenia. Hematol Oncol 31:189-96

36. Yapici O, Gunseren F, Yapici H, et al (2016) Evaluation of febrile neutropenic episodes in adult patients with solid tumors. Mol Clin Oncol 4:379-82

37. Wang XJ, Goh DYT, Dorajoo SR, Chan A (2017) The prognostic performance of adding patient-reported outcomes to the MASCC risk index to identify low-risk febrile neutropenia patients with solid tumors and lymphomas. Support Care Cancer 25:2815-22

38. Carmona-Bayonas A, Gómez J, González-Billalabeitia E, et al (2011) Prognostic evaluation of febrile neutropenia in apparently stable adult cancer patients. Br J Cancer 105:612-7

39. Carmona-Bayonas A, Jiménez-Fonseca P, Virizuela Echaburu J, et al (2015) Prediction of serious complications in patients with 
seemingly stable febrile neutropenia: validation of the Clinical Index of Stable Febrile Neutropenia in a prospective cohort of patients from the FINITE study. J Clin Oncol 33:465-71

40. Diepold M, Noellke P, Duffner U, et al (2008) Performance of interleukin-6 and interleukin-8 serum levels in pediatric oncology patients with neutropenia and fever for the assessment of lowrisk. BMC Infect Dis 8:28

41. de Bont ES, Vellenga E, Swaanenburg J, Kamps W (2000) Procalcitonin: a diagnostic marker of bacterial infection in neutropenic cancer patients with fever? Infection 28: 398-400

42. Giamarellos-Bourboulis EJ, Grecka P, Poulakou G, et al (2001) Assessment of procalcitonin as a diagnostic marker of underlying infection in patients with febrile neutropenia. Clin Infect Dis 32: 1718-25

43. Jimeno A, Garcia-Velasco A, del Val O, et al (2004) Assessment of procalcitonin as a diagnostic and prognostic marker in patients with solid tumors and febrile neutropenia. Cancer 100: 2462-9

44. Montassier E, Batard E, Gastinne T, et al (2013) Recent changes in bacteremia in patients with cancer: a systematic review of epidemiology and antibiotic resistance. Eur J Clin Microbiol Infect Dis $32: 841-50$

45. Trecarichi EM, Tumbarello M (2014) Antimicrobial-resistant Gram-negative bacteria in febrile neutropenic patients with can- cer: current epidemiology and clinical impact. Curr Opin Infect Dis 27:200-10

46. Przybylski DJ, Reeves DJ (2017) Impact of fluoroquinolone prophylaxis on documented infections in patients with febrile neutropenia. Infect Dis 50:395-8

47. Horita N, Shibata Y, Watanabe H, et al (2017) Comparison of antipseudomonal $\beta$-lactams for febrile neutropenia empiric therapy: systematic review and network meta-analysis. Clin Microbiol Infect 23:723-9

48. Cooper MR, Durand CR, Beaulac MT, Steinberg M (2011) Single-agent, broad-spectrum fluoroquinolones for the outpatient treatment of low-risk febrile neutropenia. Ann Pharmacother 45:1094-102

49. Kridel R, Delden CV, Calandra T, Marchetti O (2008) Antibiothérapie empirique lors de neutropénie fébrile. Rev Med Suisse 4:914-9

50. Talcott JA, Yeap BY, Clark JA, et al (2011) Safety of early discharge for low-risk patients with febrile neutropenia: a multicenter randomized controlled trial. J Clin Oncol 29:3977-83

51. Bitar R (2015) Utility of the Multinational Association for Supportive Care in Cancer (MASCC) risk index score as a criterion for nonadmission in febrile neutropenic patients with solid tumors. Perm J 19:37-47 